This is a repository copy of Coalitional Model Predictive Control on Freeways Traffic Networks in the Depósito de Investigación de la Universidad de Sevilla.

Version: Author Accepted Version

Citation: P. Chanfreut, J. M. Maestre and E. F. Camacho, "Coalitional Model Predictive Control on Freeways Traffic Networks," in IEEE Transactions on Intelligent Transportation Systems, doi: https://doi.org/10.1109/ TITS.2020.2994772.

To cite this publication, please use the final published version. Please check the document version above.

Copyright: (c) 2020 IEEE. Personal use of this material is permitted. Permission from IEEE must be obtained for all other uses, in any current or future media, including reprinting/republishing this material for advertising or promotional purposes, creating new collective works, for resale or redistribution to servers or lists, or reuse of any copyrighted component of this work in other works.

Takedown policy: Please contact us (idus@us.es) and provide details if you believe this document breaches copyrights. We will remove access to the work immediately and investigate your claim. 


\title{
Coalitional Model Predictive Control on Freeways Traffic Networks
}

\author{
Paula Chanfreut, José María Maestre, and Eduardo F. Camacho
}

\begin{abstract}
This paper discusses the application of coalitional model predictive control (MPC) to freeways traffic networks, where the goal is reducing the time spent by the drivers through a dynamic setting of variable speed limits (VSL) and ramp metering. The prediction model METANET is used to represent the traffic flows evolution. The system behavior and objective function lead to a non-convex and non-linear optimization problem, which can only be solved in a centralized fashion for small networks. The underlying motivation of this paper is the continued advance of clustering methods in the control of largescale and spatially distributed systems. The global freeway system is partitioned into a set of coupled sub-stretches, which in turn are assigned to the different agents involved in the control problem. These local controllers can dynamically assemble into coalitions to take coordinated measures. In this work, a top-down approach is considered: the bottom layer consists of the set of controllers that compute the VSL and ramp-metering across time; and the supervisory layer changes periodically the information exchange structure to promote coalitions of those controllers that bring greater performance to the global system. In this way, a balance is sought between optimality and efficiency. Finally, the coalitional approach is simulated on a stretch of traffic freeway where cooperation with adjacent sub-stretches is allowed.
\end{abstract}

Index Terms-Distributed model predictive control, coalitional control, control by clustering, traffic systems.

\section{INTRODUCTION}

$\mathbf{T}$ HE high increase in the number of circulating vehicles in the past decades and the ensuing congestion problems have motivated the study of control strategies for freeways. The consequences of traffic jams go beyond the drivers' time delays, as this recurring problem has also an impact on the security of roads and on the environment. An effective measure to relieve traffic jams is to perform a dynamic control of traffic flows. In this respect, previous studies have shown a good performance of model predictive controllers [1] in the field of traffic freeways at research level, e.g., [2], [3], [4]. Ramp metering [5] and variable speed limits (VSL) [6] are commonly used as traffic control signals. Other works have also used MPC for controlling reversible lanes [7] and route guidance [8].

Model predictive control (MPC) encloses a set of techniques that are built upon the same fundamental ideas [1]. Among them, there are the distributed strategies (DMPC) [9] [10], which are especially relevant nowadays given the sheer size of the systems under control and the subsequent computational demands. The basic idea is to decompose the global

The authors would like to express their gratitude for the financial support from the Spanish Training program for Academic Staff (FPU17/02653), the Spanish MINECO project C3PO (ref: DPI2017-86918-R), and the European Research Council Advanced Grant OCONTSOLAR (ref: SI-1838/24/2018).

P. Chanfreut, J.M. Maestre and E.F. Camacho are with the Department of Systems and Automation Engineering, University of Seville, Spain. problem into smaller subproblems, each of them assigned to a controller [11]. In the context of DMPC, the idea of coalitional MPC rose as a response to the increasing interest in practical techniques for controlling large-scale systems, considering performance, complexity, and coordination costs. Coalitional control is a novel set of techniques that go hand in hand with game theory [12]-[15], and that aims to optimize the degree of communication and cooperation between controllers across time, encouraging local controllers to aggregate into time-varying clusters, hereon coalitions. The idea of dynamic clusters of controllers is taking a strong role in the control of multi-agent networked systems [16]-[19]. In particular, in [20] the main coalitional MPC concepts and challenges are discussed. The aim is to resort to information exchange and cooperation mechanisms only when it provides significant benefits for the system performance, thus allowing us to work with a structure that is adapted to the variable coupling conditions over time. Previous works on the field show very promising results of this recent control strategy, e.g., in [20] a simulation on a smart-grid example is presented, and in [21] coalitional MPC is applied to irrigation canals.

In traffic systems, the number of control and state variables (densities, mean speed, and queues along the network) and the high communication overhead associated with centralized control restricts its use to small networks. Nowadays, the computation of the control signals is usually made based on local measures. However, greater performance could be achieved considering the global impact of local measures and coordinating the traffic signals [22], [23]. Distributed strategies for traffic freeways have been studied in [24], [25]. In this context, the roads are divided into interacting stretches governed by different controllers. The strong couplings existing between each section in a traffic network makes the cooperation between controllers determinant for global performance. Additionally, congestion problems tend to concentrate at particular times of the day and arise at scattered locations. The spatially and timely distributed events that characterize traffic systems, together with the complexity of a centralized problem, make coalitional control particularly worthy of study in this field. Moreover, the traffic problem we address shares certain commonalities with the flow regulation that is done in [21], which is quite an incentive to think that the results already seen in this case can be extrapolated for a traffic example.

In this work, the coalition formation is based on a top-down architecture, where a supervisory layer receives information from the controllers and sets dynamically the communication topology. The controllers at the bottom-layer change the values of the speed limits and measure the incoming flows from the 
on-ramps. The controls operating ranges are discretized and the actions are optimized using the Genetic Algorithm [26].

Hereafter, the article is organized as follows. First, in Section II, the traffic model METANET is introduced. In Section III, an introduction to coalitional MPC is provided. Subsequently, Section IV focuses on the application of the coalitional approach to the freeway traffic problem. Finally, in Section V, our simulation results are presented.

\section{MACROSCOPIC TRAFFIC MODEL METANET}

To predict the evolution of the traffic in the network, we will use the METANET model [27], which uses a fluidlike macroscopic representation of traffic flows. It is a second-order model whose equations can easily be extended to incorporate the effect of control actions such as VSL [2] and ramp metering [28]. However, note that the control methods studied in this work can be applied to other macroscopic models as well, such as the CTM [29] or any of its variants.

METANET fundamental diagram (see Figure 1) provides us with the relation between traffic flow (veh/time) and density (veh/length), and so implicitly specifies also the mean speed. It can be seen that there is a critical density, hereafter denoted as $\rho_{\text {crit }}$, from which the flow begins to drop, reaching zero when the density is $\rho_{\text {jam }}$. The critical density sets a clear boundary regarding how an increment of density affects the circulating traffic flow. From now on, free-flow conditions will refer to situations when the densities are lower than $\rho_{\text {crit }}$, and congested conditions will be used otherwise.

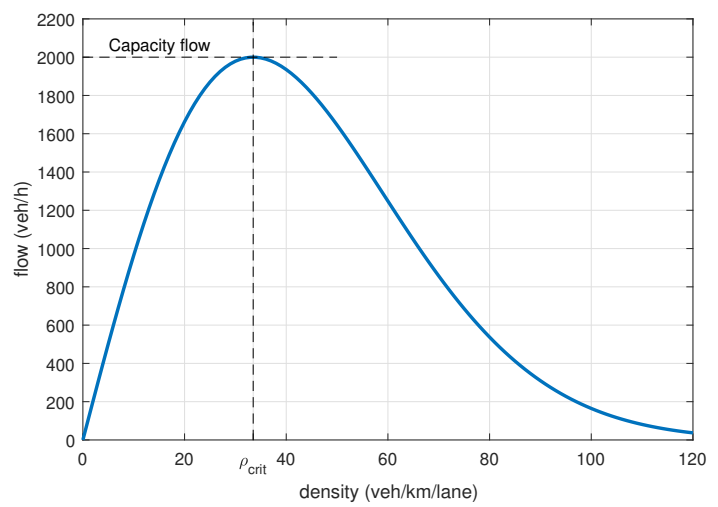

Fig. 1. METANET Fundamental Diagram.

METANET discretizes the global network into a set of links $\mathcal{M}=\{1, \ldots, M\}$, which in turn are composed of a set of segments $\mathcal{I}_{m}=\left\{1, \ldots, I_{m}\right\}$. The latter implies working with a set of spatially distributed variables, which given the nature of traffic networks, will be strongly coupled. Each time instant $k$, the state of each segment $i \in \mathcal{I}_{m}$ of a given link $m \in \mathcal{M}$ is defined by its density $\rho_{m, i}(k)$ and its mean speed $v_{m, i}(k)$. In case that there is an on-ramp in the segment, its state is extended with the variable $w_{m, i}(k)$, which represents the queue of cars waiting to access the main road.

For simplicity, the METANET model equations presented here assume that all segments grouped in a link are equal, and thus their model parameters take the same values.

\section{A. Mainline/Queue Conservation Equations}

Let $q_{m, i}(k)$ be the outflow of segment $i$ in link $m$, which, by the boundary conditions, becomes an inflow for the segment downstream. Likewise, $q_{\mathrm{ramp}_{m, i}}(k)$ represents the flow that enters the main road from an on-ramp at the beginning of segment $i$. Assuming that the off-ramp flow can be expressed proportionally to $q_{m, i}(k)$ using a split ratio $\beta_{m, i}(k)$, the conservation equations are:

$$
\begin{array}{r}
\rho_{m, i}(k+1)=\rho_{m, i}(k)+\frac{T}{\lambda_{m} L_{m}}\left(q_{m, i-1}(k)+q_{\mathrm{ramp}_{m, i}}(k)\right. \\
\left.-\left(1+\beta_{m, i}(k)\right) q_{m, i}(k)\right), \\
(1) \\
w_{m, i}(k+1)=w_{m, i}(k)+T\left(d_{m, i}(k)-q_{\mathrm{ramp}_{m, i}}(k)\right), \quad(2)
\end{array}
$$

where $\lambda_{m}$ and $L_{m}$ denote respectively the number of lanes and length of the segments in the freeway link $m$, and $T$ represents the model sample time.

Equations (1) and (2) model the vehicles density and queue length evolutions in each $i \in \mathcal{I}_{m}$, respectively. Note that (2) is dropped for all the segments without on-ramps. Likewise, $q_{\mathrm{ramp}_{m, i}}(k)=0$ in the latter case, and $\beta_{m, i}(k)=0$ for all segments without off-ramps. Figure 2 shows schematically all the above-mentioned variables.

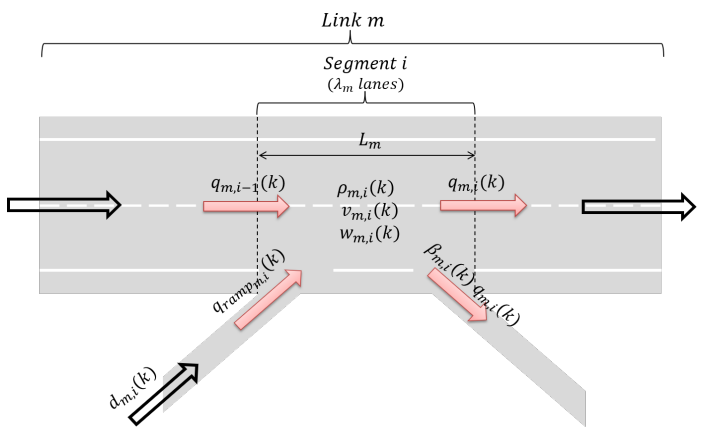

Fig. 2. Freeway link scheme.

\section{B. Speed dynamics}

The mean speed update equation is defined as

$$
\begin{aligned}
v_{m, i}(k+1)=v_{m, i}(k) & +\underbrace{\frac{T}{\tau_{m}}\left(V\left(\rho_{m, i}(k)\right)-v_{m, i}(k)\right)}_{\Delta v_{m, i}^{\mathrm{r}}(k)} \\
& +\underbrace{\frac{T}{L_{m}} v_{m, i}(k)\left(v_{m, i-1}(k)-v_{m, i}(k)\right)}_{\Delta v_{m, i}^{\mathrm{c}}(k)} \\
& -\underbrace{\frac{\vartheta_{m} T}{\tau_{m} L_{m}} \frac{\rho_{m, i+1}(k)-\rho_{m, i}(k)}{\rho_{m, i}(k)+\kappa_{m}}}_{\Delta v_{m, i}^{\mathrm{a}}(k)},
\end{aligned}
$$

where $\tau_{m}, \vartheta_{m}, \kappa_{m}$ are model parameters for all segments in link $m$. As can be seen, the speed update is governed by three terms: 
- $\Delta v_{m, i}^{\mathrm{r}}(k)$ is the relaxation term, which considers the tendency of the drivers to achieve their desired speed, defined as

$$
\begin{aligned}
V\left(\rho_{m, i}(k)\right)= & \min \left[\alpha V S L_{m, i}(k),\right. \\
& \left.v_{\text {free }, m} \exp \left[-\frac{1}{a_{m}}\left(\frac{\rho_{m, i}(k)}{\rho_{\text {crit }, m}}\right)^{a_{m}}\right]\right],
\end{aligned}
$$

where $a_{m}$ is a model parameter and $V S L_{m, i}(k)$ are the variable speed limits, which will be control variables along this paper, and $v_{\text {free, } m}$ denotes the vehicles mean speed when the traffic situation is that of free flow. The parameter $\alpha$ is introduced to consider that drivers desired speed tends to exceed the speed limits displayed.

- $\Delta v_{m, i}^{\mathrm{c}}(k)$ is the convection term, which expresses the influence of the situation upstream.

- $\Delta v_{m, i}^{\mathrm{a}}(k)$ is the anticipation term, which adds the effect of a higher or lower density downstream.

Equation (3) can be extended to take into account the speed decrease resulting from merging phenomena. If the segment $(m, i)$ has an on-ramp, the following term is added to the mean speed update equation

$$
\Delta v_{m, i}^{\mathrm{m}}(k)=-\frac{\delta_{m} T q_{\mathrm{ramp}_{m, i}}(k) v_{m, i}(k)}{L_{m} \lambda_{m}\left(\rho_{m, i}(k)+\kappa_{m}\right)},
$$

where $\delta_{m}$ is also a model parameter. Using the expressions above, the flow transfer between segments is finally defined as

$$
q_{m, i}(k)=\lambda_{m} \rho_{m, i}(k) v_{m, i}(k) .
$$

1) Boundary conditions: The following assumptions are considered [2]:

- The mean speed of the mainline source segment is equal to the speed of the first segment. That is, in (3), we will use $v_{1,0}(k)=v_{1,1}(k)$ for all $k$. Note that this assumption cancels the convection term for the first segment of road.

- The conditions at the exit of the main road are those of free flow. In particular, denoting the density downstream the last segment as $\rho_{M+1,1}$, we assume

$$
\rho_{M+1,1}(k)=\left\{\begin{array}{cl}
\rho_{M, I_{M}}(k) & \text { if } \rho_{M, I_{M}}(k)<\rho_{\text {crit }, m} \\
\rho_{\text {crit }, m} & \text { if } \rho_{M, I_{M}}(k) \geq \rho_{\text {crit }, m}
\end{array}\right.
$$

\section{On-ramp flows}

Additionally, for the on-ramp flows it holds

$$
\begin{array}{r}
q_{\mathrm{ramp}_{m, i}}=r_{m, i}(k) \cdot \min \left[d_{m, i}(k)+\frac{w_{m, i}(k)}{T}, Q_{\mathrm{ramp}_{m, i}},\right. \\
\left.Q_{\mathrm{ramp}_{m, i}}\left(\frac{\rho_{\mathrm{jam}, m}-\rho_{m, i}(k)}{\rho_{\mathrm{jam}, m}-\rho_{\mathrm{crit}, m}(k)}\right)\right]
\end{array}
$$

where $Q_{\mathrm{ramp}_{m, i}}$ is the on-ramp capacity, $r_{m, i}(k) \in[0,1]$ is the on-ramp metering rate, and $d_{m, i}(k)$ is the external flow of vehicles received by the on-ramp. Therefore, if $r_{m, i}(k)=1$, the flow that enters the main road from the on-ramp is defined as the minimum between the number of cars available (considering those that are already in queue and those received that time instant), the maximum flow that the mainline can admit due to its traffic condition, and the on-ramp capacity. Likewise, if $r_{m, i}(k)=0$, the access to the main road will be completely banned.

In addition to the on-ramps, the mainstream origin is another entry point of vehicles where a queue might be formed. In this case, the conditions of the first segments to admit the arriving cars are crucial.

1) Mainstream origin: The origin of the road is modeled using the on-ramp queues equation (2), together with a modified version of (8). Let us denote the mainstream origin with the subindex 0 , then

$$
w_{0}(k+1)=w_{0}(k)+T\left(d_{0}(k)-q_{0}(k)\right)
$$

and

$$
\begin{aligned}
& \rho_{1,1}(k+1)=\rho_{1,1}(k)+ \\
& \frac{T}{\lambda_{1} L_{1}}\left(q_{0}(k)+q_{\mathrm{ramp}_{1,1}}(k)-\left(1+\beta_{1,1}(k)\right) q_{1,1}(k)\right),
\end{aligned}
$$

where

$$
q_{0}(k)=\min \left[d_{0}(k)+\frac{w_{0}(k)}{T}, q_{\lim }(k)\right] .
$$

The variable $d_{0}(k)$ represents the external demand at the beginning of the road. Moreover, $q_{\lim }(k)$ is defined as

$$
q_{\lim }(k)=\lambda_{1} v_{\lim }(k) \rho_{\text {crit }, 1}\left[-a_{1} \ln \left(\frac{v_{\text {lim }, 1}(k)}{v_{\text {free }, 1}}\right)\right]^{1 / a_{1}}
$$

if $v_{\lim , 1}<V\left(\rho_{\text {crit }, 1}\right)$, and $q_{\lim }(k)=\lambda_{1} V\left(\rho_{\text {crit }, 1}\right) \rho_{\text {crit }, 1}$ otherwise, with

$$
v_{\lim }(k)=\min \left[V S L_{1,1}(k), v_{1,1}(k)\right] .
$$

\section{INTRODUCTION TO COALITIONAL MPC}

In this section, a brief introduction to coalitional MPC based on [12] and [20] is presented.

Let us consider a global system composed of a set $\mathcal{N}=$ $\{1, \ldots, N\}$ coupled subsystems, each of them assigned to a controller and interconnected by communication links. Let the discrete-time dynamics of subsystem $i \in \mathcal{N}$ be expressed as

$$
x_{i}(k+1)=f\left(x_{i}(k), u_{i}(k)\right)+\sum_{l \neq i} g\left(x_{l}(k), u_{l}(k)\right),
$$

where $x_{i} \in \mathbb{R}^{n_{i}}$ is the subsystem state, $u_{i} \in \mathbb{R}^{m_{i}}$ represents its input vector, and $\sum_{l \neq i} g\left(x_{l}(k), u_{l}(k)\right)$ is the disturbance received by agent $i$.

The data network of the system will be described by an undirected graph denoted as $\mathcal{G}=(\mathcal{N}, \mathcal{L})$, where $\mathcal{N}$ represents the set of agents and $\mathcal{L}$ the set of physical communication links between them. That is,

$$
\mathcal{L} \subseteq \mathcal{L}^{\mathcal{N}}=\left\{\ell_{i, l} \mid\{i, l\} \subseteq \mathcal{N}, i \neq l\right\} .
$$

In coalitional control, different communication topologies $\Lambda$ can be used across time, i.e., the network topology is defined by the enabled links $(\Lambda \subseteq \mathcal{L})$. As consequence, the set of controllers $\mathcal{N}$ is divided into different disjoint communication components or coalitions as

$$
\mathcal{N} / \Lambda=\left\{C_{1}, C_{2}, \ldots, C_{|\mathcal{N} / \Lambda|}\right\},
$$


where $C_{j}$ for all $j=1, . .,|\mathcal{N} / \Lambda|$ denote coalitions of cooperating controllers, with $\cup_{C_{j} \in \mathcal{N} / \Lambda} C_{j}=\mathcal{N}$. The number of coalitions $|\mathcal{N} / \Lambda|$ will be an integer number ranging from 1 (centralized case) to $N$ (fully decentralized case).

Inside each coalition, controllers share information and take joint actions to determine their optimal control law. Dynamically, each $C_{j}$ can be seen as a single entity, for which $x_{C_{j}}(k)$ and $u_{C_{j}}(k)$ are the aggregate of the states and inputs of the subsystems grouped in $C_{j}$, that is,

$$
x_{C_{j}}(k+1)=f\left(x_{C_{j}}(k), u_{C_{j}}(k)\right)+\sum_{l \neq j} g\left(x_{C_{l}}(k), u_{C_{l}}(k)\right)
$$

where $x_{C_{j}}(k)=\left[x_{i}(k)\right]_{i \in C_{j}}$ and $u_{C_{j}}(k)=\left[u_{i}(k)\right]_{i \in C_{j}}$. The term $g\left(x_{C_{l}}(k), u_{C_{l}}(k)\right)$ is the state disturbance that results from mutual interactions with other coalitions $C_{l} \in$ $\mathcal{N} / \Lambda \backslash\left\{C_{j}\right\}$. The dimmensions above are defined accordingly, i.e., $x_{C_{j}}(k) \in \mathbb{R}^{\sum_{i \in C_{j}} n_{i}}, u_{C_{j}}(k) \in \mathbb{R}^{\sum_{i \in C_{j}} m_{i}}$ and $g\left(x_{C_{l}}(k), u_{C_{l}}(k)\right) \in \mathbb{R}^{\sum_{i \in C_{j}} n_{i}}$.

Each coalition $C_{j}$ solves locally its control problem to maximize the overall performance of subsystems $i \in C_{j}$. In particular, each coalition solves an MPC optimization problem formulated as follows:

$$
\begin{array}{cl}
\min _{\mathbf{u}_{C_{j}}(k)} & J_{C_{j}}\left(x_{C_{j}}(k), \mathbf{u}_{C_{j}}(k)\right) \\
\text { s.t. } & (17), \\
& u_{C_{j}}(k+n) \in \mathcal{U}_{C_{j}}, \\
& x_{C_{j}}(k+n+1) \in \mathcal{X}_{C_{j}}, \\
& x_{C_{j}}(k)=x_{C_{j}, 0}, \\
& \forall n=0, \ldots, N_{\mathrm{p}}-1 .
\end{array}
$$

where $J_{C_{j}}(\cdot)$ represents the coalition objective along a prediction horizon $N_{\mathrm{p}}$. The control variable $\mathbf{u}_{C_{j}}(k)$ is the sequence vector $\mathbf{u}_{C_{j}}(k)=$ $\left[\begin{array}{llll}u_{C_{j}}(k) & u_{C_{j}}(k+1) & \cdots & u_{C_{j}}\left(k+N_{\mathrm{p}}-1\right)\end{array}\right]^{\mathrm{T}^{j}} \in$ $\mathbb{R}^{N_{\mathrm{p}} \sum_{i \in C_{j}} m_{i}}$. Additionally, $\mathcal{X}_{C_{j}}$ and $\mathcal{U}_{C_{j}}$ represent respectively the constraints sets imposed on the state and inputs of coalition $j$, and $x_{C_{j}, 0}$ is its initial state.

The goal of coalitional control is optimizing the overall performance while reducing the communication burden associated to cooperation by enabling and disabling the links according to their contribution to the control objectives. The global problem can be posed as

$$
\begin{aligned}
\min _{\Lambda(k), \mathbf{u}(k)} & \sum_{C_{j} \in \mathcal{N} / \Lambda(k)} J_{C_{j}}\left(x_{C_{j}}(k), \mathbf{u}_{C_{j}}(k), \Lambda(k)\right)+N_{\mathrm{p}} c_{l}|\Lambda(k)| \\
\text { s.t. } & (17) \\
& u_{C_{j}}(k+n) \in \mathcal{U}_{C_{j}} \\
& x_{C_{j}}(k+n+1) \in \mathcal{X}_{C_{j}}, \\
& x_{C_{j}}(k)=x_{C_{j}} \\
& \forall j=1, \ldots,|\mathcal{N} / \Lambda(k)| \\
& \forall n=0, \ldots, N_{\mathrm{p}}-1 .
\end{aligned}
$$

where the global performance index is defined as the sum of the communication components objectives. The cost of enabling a link is represented as $c_{l}$, and $|\Lambda(k)|$ is the number of active links in topology $\Lambda(k)$. For simplicity, we will assumme that $c_{l}$ takes a constant and equal value for all links. The centralized control variable $\mathbf{u}(k)$ aggregates the coalitions control actions sequences, i.e., $\mathbf{u}(k)=\left[\mathbf{u}_{C_{j}}(k)\right]_{C_{j} \in \mathcal{N} / \Lambda(k)}$.

\section{COALITIONAL MPC APPROACH FOR TRAFFIC FREEWAYS}

For the freeway traffic system, the subsystems will be the links of freeway into which the global road is partitioned. For simplicity, our system will consist of a freeway corridor of $M$ links with on-ramp/off-ramps, i.e., $\mathcal{N}=\{1, \ldots, M\}$. As described in Section II, the freeway links will be also divided into segments for a better representation of the traffic evolution within them. In this problem, we consider that sections of road where the traffic is not directly monitored can also constitute single subsystems. In these cases, and following the formulation of coalitional control, we will also consider a local control entity, whose objective is not to optimize any sequence of inputs but to collect and exchange local data when corresponds.

Generally, we aim at solving problem (19) applied to the traffic case. However, this problem belongs to the class of NP-complete [30], which restricts its applicability for realtime control to small networks unless some simplifications are introduced. In this work, we consider a double sample rate strategy, in which switchings of coordination mode are only periodically allowed. Two architectures are commonly used for the topology optimization [20]: top-down and bottom-up, being the former the one we will use here. In particular, we have implemented a supervisory layer that periodically selects a certain topology amongst a set of predefined possibilities, aiming at enhancing the global performance. Figure 3 shows a graphical description of the scheme used.

Once a topology $\Lambda$ is set, the global system is controlled according to partition $\mathcal{N} / \Lambda=\left\{C_{1}, C_{2}, \ldots, C_{|\mathcal{N} / \Lambda|}\right\}$ for a certain amount of time steps. In this context, each coalition $C_{j}$ will be associated with a subset of sections of freeway and will perform in a decentralized manner from the rest of the system, i.e., there is no communication within clusters. The timevarying communication structure will allow the local entities to aggregate into groups and work together for stretches of variable length.

Without loss of generality, we consider that the control objective is to minimize the total time spent (TTS) by the drivers on the road while reducing communication and coordination costs. The TTS is an index that has been widely used to define the objective function of MPC controllers in the field of traffic freeways, e.g., [2], [25], [31]. Additional measures to assess traffic behavior may also suit better for other traffic scenarios, e.g., the density balance [32], which is used in urban traffic problems to assess vehicles distribution in the network, and the service of demand [33], which focuses on the ability of the network to absorb the external inflows.

\section{A. Sampling rates}

Three different sampling rates are considered. Firstly, $T$ is the model sample time (i.e., the simulation step size), and will 


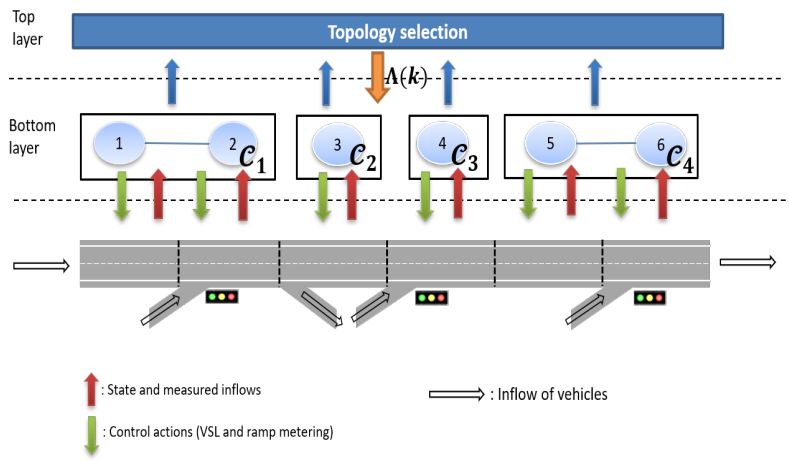

Fig. 3. Scheme of the top-down approach for $|\mathcal{N} / \Lambda|=4$ and 6 freeway links.

be chosen considering the numerical stability condition for the model, that is, $T v_{\text {free }_{m}} \leq L_{m}$. Secondly, $T_{\mathrm{u}}>T$ is used for the low-level controller step size, and it is defined as the time interval for which each control law is active and for which the control actions remain constant. Finally, changes in topology will be set each $T_{\text {top }}$ minutes, with $T_{\text {top }}>T_{\mathrm{u}}$.

\section{B. Low-level controller}

At the bottom layer, each cluster forms an independent entity where the traffic signals are set cooperatively to improve the traffic performance along all sections of freeway associated to the corresponding local entities. The control variables considered along this work are the VSL and the on-ramp metering. The former comes into play through the relaxation term in the speed dynamics (3), whose alteration will consequently affect the flow transfer in the road given (6). On the other hand, the on-ramp metering rates are used to regulate the flow of vehicles that enters into the mainline. In particular, the optimization problem of each coalition can be posed as:

$$
\begin{aligned}
\min _{\mathbf{u}_{C_{j}}(k)} & J_{C_{j}}\left(x_{C_{j}}(k), \mathbf{u}_{C_{j}}(k)\right) \\
\text { s.t. } & (1)-(8) \\
& V S L_{\min } \leq V S L_{m, i}(k+n) \leq V S L_{\max }, \\
& 0 \leq r_{m, i}(k+n) \leq 1, \\
& x_{C_{j}}(k)=x_{C_{j}, 0}, \\
& \forall(m, i) \in C_{j}, \\
& \forall n=0, \ldots, N_{\mathrm{p}}-1 .,
\end{aligned}
$$

where the state and inputs are defined as

$$
\begin{aligned}
& x_{C_{j}}(k)=\left[\rho_{m, i}(k), w_{m, i}(k), v_{m, i}(k)\right]^{\mathrm{T}}, \\
& u_{C_{j}}(k)=\left[V S L_{m, i}(k), r_{m, i}(k)\right]^{\mathrm{T}}, \\
& \forall(m, i) \in C_{j} \text { and } j=1, \ldots,|\mathcal{N} / \Lambda| .
\end{aligned}
$$

The pair $(m, i) \in C_{j}$ indicates that the freeway link $m$ with its correspoding segment $i$ belongs in the coalition $C_{j}$. The lower and upper bounds imposed on the control actions are represented by $r_{\min }$ and $r_{\max }$ for the ramp metering, and $V S L_{\min }$ and $V S L_{\max }$ for the VSL, respectively. Setting $r_{\min }>0$ helps us to ensure that there is always a certain flow from the on-ramp allowed to enter the main road. The use of
$V S L_{\min }>0$ is in line with the latter, but in this case is to avoid blockages in the mainstream flow.

In our simulations, we have considered that the controllers aim at reducing the total time spent by the drivers, so that the TTS is the most important term in the coalitions objective function, i.e.,

$$
\begin{aligned}
& J_{\mathrm{TTS}}(k)= \\
& T \sum_{j=0}^{N_{\mathrm{p}}-1} \sum_{(m, i) \in C_{j}}\left(L_{m} \lambda_{m} \rho_{m, i}(k+j)+w_{m, i}(k+j)\right),
\end{aligned}
$$

where the densities and queues evolution are defined by (1) and (2), respectively. The first term in $J_{\text {TTS }}$ represents the total travel time in the main road (TTT), while the second one considers the total waiting time on entrance queues (TWT). Moreover, a term to penalize sudden changes in the control signals has been added to (22). For the case of $r_{m, i}$, it is defined as

$J_{\Delta r}(k)=\epsilon \sum_{(m, i) \in C_{j}} \sum_{n=0}^{N_{\mathrm{p}}-1}\left\|r_{m, i}(k+n+1)-r_{m, i}(k+n)\right\|^{2}$,

where $\epsilon$ is a constant weight factor. The coalitions cost function has been defined as the sum of the objectives presented above.

Problem (20) has been approached from a distributed perspective. In particular, within each communication component $C_{j}$, we have implemented the FC-MPC algorithm (Feasible Cooperation-Based MPC) [34]. Under this framework, the control entities enter a negotiation process in which information is iteratively exchanged and in which all controllers optimize a common coalitional objective. In return, the complexity of the control problem and the dependency of non-local data increase in comparison to a decentralized approach. The latter will notably depend on the size of the coalition, i.e., the number of assembled controllers, and the number of control variables to be coordinated. Note that in case of a coalition between subsystems with and without control inputs, there may be no need for negotiation. In those cases, problem (20) can be solved after sharing local data, i.e., densities $\rho_{m, i}$, mean speeds $v_{m, i}$ and, where appropriate, waiting queues $w_{m, i}$. In this context, the wider knowledge on the neighbors' information and their consideration in the objective function commonly lead to an enhanced performance.

To avoid the interdependency of the coalitions' MPC problems, the boundary conditions given in Subsection II.B are applied accordingly in the corresponding sub-stretches of road and input flows are estimated when necessary. In particular, the following assumptions are considered:

Assumption 1: The state of the all $(m, i) \in C_{j}$ is fully available for coalition $j$, that is, the densities, mean speeds and queues length can be measured every time instant $k$ for all the segments.

Assumption 2: External inflows $d_{m, i}(k)$ are assumed to be known, being its estimation beyond the scope of this work.

Assumption 3: Each controller can measure the inflow into its freeway link. Note that the controllers in charge of any link $m>1$ have to estimate the outflow of the link upstream, 
i.e., $q_{m-1, I_{m-1}}(k)$, as its real evolution cannot be known beforehand and depends on the decisions taken by others controllers. If $m$ and $m-1$ form a coalition, the inflow estimation will be made considering the measurements of $q_{m-2, I_{m-2}}(k)$.

Remark 1: A typical concern in distributed control systems is that of tolerance to failures. A common approach is to exploit the inherent redundancy of distributed control to reconfigure the network as in [35]-[37] and to let controllers detect and robustify their computations against noncompliant subsystems [38]. In coalitional control, the dynamic structure of the coalitional controller allows dealing with communication failures between agents. In particular, if $\ell_{m, n}$ is a faulty link connecting agents $m$ and $n$, then, the system could be operated with any topology in which link $\ell_{m, n}$ is disabled, i.e., agents $m$ and $n$ belonging to different coalitions.

\section{Prediction horizon setting}

Considering the conservation equations in the global stretch, we can conclude that for equal inflows a decrease of the global TTS is caused by an increase of the output flow. Given the geographically dispersed nature of the system, the impact of the control actions on distant locations might not be visible after some time. When using the TTS as objective function, the number of samples times for which the performance is predicted should be large enough to perceive the effect of the different VSLs or ramp metering rates on the output flow. Otherwise, it might lead to an inaccurate choice of the traffic signals. See [5] for more details regarding the latter conclusion.

In other words, $N_{\mathrm{p}}$ should be larger than the regular driving time through the stretch of road under control. Note that this is not to say that different control actions would not have different effects on the behavior of the system, but that changes inside the network may not be perceived in the TTS function (e.g., different vehicles distribution inside the network). The latter represents a problem in a top-down coalitional control scheme, as the supervisory layer should take into account the global effect of all the spatially distributed controls in the network.

Moreover, if we assume that each controller is in charge of a sub-stretch of road, the mergers between them will lead to an optimization of a longer sub-stretch. The latter may have two sides: their cooperation can simultaneously improve the performance (expected consequence), but also if $N_{\mathrm{p}}$ is set shorter than the typical travel time through the aggregated sub-stretches, their jointly calculated control actions may be misleading. It also entails that $N_{\mathrm{p}}$ becomes an important parameter in comparing, under the same conditions, the performance of joined/disjoined controllers.

\section{Upper layer}

The criterion used for the forming of coalitions is crucial for both system optimality and communication costs incurred. In [12] and [20], an index that weights both system performance and communication costs is used. In this work, the problem of switchings between topologies is modeled as a decision problem that is solved periodically at the top layer. Considering our control objective, the communication modes selected across time should be those that bring greater reductions of the global TTS during their operation period at the minimum communication costs. To avoid the computation demands of an optimization-based topology selection procedure, a heuristic approach where the top layer determines the switchings of coordination modes according to a heuristic criterion is proposed in this paper, while the VSLs and rampmetering are optimized in a distributed (coalitional) manner at the bottom layer. Due to the dynamical couplings, the optimality of local actions is influenced by the state and traffic signals imposed by the rest of agents in the network. In this context, the benefits of greater coordination strongly depend on the coupling conditions between the subsystems. Considering the latter, we will foster the use of coordination mechanisms among those controllers associated with stretches of road where the traffic situation is (expected to be) more critical. In particular, let $\ell$ denote any link of the communication network and $C_{\ell}$ be the coalition resulting from enabling link $\ell$. Then, the links relevance have been evaluated according to the vehicles distribution along the road and the expected inflows during the next interval $T_{\text {top }}$ as follows

$$
\begin{aligned}
J_{\ell}(k)= & \sum_{(m, i) \in C_{\ell}}\left(L_{m} \lambda_{m} \rho_{m, i}(k)+w_{m, i}(k)\right. \\
& \left.+T \sum_{j=0}^{T_{\text {top }} / T-1} q_{\text {ramp }_{m, i}}(k+j)\right),
\end{aligned}
$$

where the last term considers the influence of the future vehicles entries and thus allows us to anticipate possible traffic congestions when their effects are not visible at the time of the topology selection. Finally, the function we have used to assess the performance of each topology is

$$
J_{\Lambda}(k)=-\sum_{\ell \in \Lambda} J_{\ell}(k)+T_{\text {top }} c_{l}|\Lambda| .
$$

Then, each $T_{\text {top }}$ minutes, the local control entities communicate real-time traffic data to the top layer, i.e., local densities, queues, and on-ramp flows estimations, and subsequently the topologies are compared through a rating procedure based on function (25). The communication mode that results in the minimum value of (25) is selected and the corresponding clusters are formed.

Remark 2: One of the challenging issues of the coalitional approach is the combinatorial explosion with the problem size. As it is well known, in combinatorial optimization problems it is only possible to obtain the optimal solution in a general way for very specific types of problems, e.g., submodular functions. Otherwise, it is necessary to resort on suboptimal methods that can be computed within the time step length. For example, one option is to compute the Shapley value as a measure of the relevance of the agents and then choose a topology accordingly, as it is done for large-scale problems in [39], [40]. It must be noted that the computation of this value can be approximated with arbitrary accuracy in polynomial time by randomized methods as [41]. Alternatively, other relevance measures such as the PageRank can be also considered as 
it is done in [42]. Again, there are efficient methods to compute the PageRank efficiently in a distributed fashion [43]. The approach followed here is aligned with the previously mentioned methods, because it considers a relevance indicator as well for each section of the road, which is the number of current and incoming cars. In this way, it is possible to identify which sections are more relevant from the viewpoint of coalition formation. Likewise, other alternatives that can be considered to reduce computation burden in a general way, e.g., the implementation of a sequential procedure to guide the activation/deactivation of the communication links in a greedy fashion.

\section{E. Search for the optimum}

The optimal sequences of VSL and ramp metering are searched using the Genetic Algorithm (GA) [26]. The GA is an optimization procedure involving iterative evaluations of a set of candidate solutions, the so-called population, which evolves based on natural selection and evolutionary genetics. In particular, from an initial population, different generations are derived using the best found candidate solutions and new ones resulting from mutation and recombination of promising options. The GA represents an efficient optimization method to deal with nonlinear objective functions with many local minima and with mixed-integer variables. In particular, see [44][47] for examples of its application in the field of traffic control.

In this paper, we use the GA for solving the optimization problems at the bottom layer. When working in coalitions, each controller uses the algorithm within the negotiation process of the FC-MPC to jointly determine the best sequences of the coalition's traffic signals. In this respect, we consider both the discrete nature of the VSL and the temporal restriction on the signals, i.e., the implemented controls remain active for a period $T_{\mathrm{u}}$. Additionally, we consider a maximum change per time instant to reduce the search space for the optimal solution. In particular, for all time instant $k_{\mathrm{u}}=p T_{\mathrm{u}} / T$ where $p$ is a non-negative integer, we will consider

$$
\begin{aligned}
& \left|V S L\left(k_{\mathrm{u}}+n\right)-V S L\left(k_{\mathrm{u}}+n+1\right)\right| \leq \Delta_{\max }^{\mathrm{VSL}}, \\
& \left|r\left(k_{\mathrm{u}}+n\right)-r\left(k_{\mathrm{u}}+n+1\right)\right| \leq \Delta_{\max }^{\mathrm{r}}, \\
& \forall n=0, \ldots,\left(N_{\mathrm{p}}-1\right) / T_{\mathrm{u}},
\end{aligned}
$$

where $\Delta_{\max }^{\mathrm{VSL}}$ and $\Delta_{\max }^{\mathrm{r}}$ denote the maximum difference in the control signals between two consecutive controller step times. These temporal restrictions on the VSL are usually considered for security reasons. In case of the ramp metering, it is consistent if the value set for $\Delta_{\max }^{\mathrm{r}}$ allows the adjustment of $r$ in a sufficiently short time to deal with the changes of demand at the onramps. Note that all of the recombination and mutation operations should take into account these constraints when acting on candidate solutions.

\section{Simulation Results}

In order to illustrate the coalitional MPC approach, we will now simulate it with the benchmark network shown in Figure 4. It consists on a freeway corridor of $15 \mathrm{~km}$ with three metered on-ramps, three speed limits, and one offramp. A division of the global road stretch into six freeway links and 15 segments (1 km/segment) is considered. For the implementation of the METANET model, we follow [2]. Likewise, the network parameters has been set as in [48] and are given in Table I. Further details about the system are provided in Table II.

The metered on-ramps and VSL distributions have been set following [2] and [49], where the authors show that speed controlled zones previous to on-ramps entries can help to reduce the TTS. Additionally, they highlight the impact of a coordinated decision on these control variables. In the segments without VSL or on-ramp metering, the traffic is not directly monitored by the controllers. The spatial constraints to be imposed given the actuation length of each speed limit have also been added to the controllers' problems.

Figure 5 shows the communication links between controllers. The network topologies considered by the top layer are: $\{\emptyset\},\{I\},\{I I\},\{I I I\},\{I V\},\{V\}$, $\{I I, I V\},\{I I, V\},\{I, I I, I V\},\{I, I I, V\},\{I, I I, I I I\}$, $\{I I, I I I, I V\},\{I, I I, I I I\}$.

Following the discussion in Section IV.D, the prediction horizon used by the coalitional controllers has been adapted according to the size of the sub-stretch of road considered. In particular, when the section of road does not exceed $3 \mathrm{~km}$ in length, $N_{\mathrm{p}}$ is set to $2 T_{\mathrm{u}}$; when it is between $3 \mathrm{~km}$ and $5 \mathrm{~km}$, $N_{\mathrm{p}}$ is extended to $3 T_{\mathrm{u}}$ minutes; and when it is over $5 \mathrm{~km}$, we use a prediction horizon of $4 T_{\mathrm{u}}$ minutes.

The constraints on queues are soft. In particular, we have added to the objective function the following term

$$
J_{L}(k)=\zeta \sum_{m, i} \delta_{w_{m, i}}(k)\left\|L_{\mathrm{ramps}}-w_{m, i}(k)\right\|^{2},
$$

where $L_{\text {ramps }}$ is the maximum number of cars in the queues, and $\zeta$ is a constant that has been set at 0.01 in our simulations. The variable $\delta_{w_{m, i}}$ is defined as

$$
\delta_{w_{m, i}}(k)=\left\{\begin{array}{ccc}
1 & \text { if } & w_{m, i}(k) \geq L_{\mathrm{ramps}} \\
0 & & \text { otherwise }
\end{array}\right.
$$

Remark 3: Note that an estimation of the main road inflow is needed in case of mid-clusters of controllers. In particular, in the simulations shown here, the coalitions' MPC problems are solved considering the following: (i) external inflows are assumed to be known, i.e., entries from the origin and onramps (see Assumption 2); (ii) the inflow from the main road

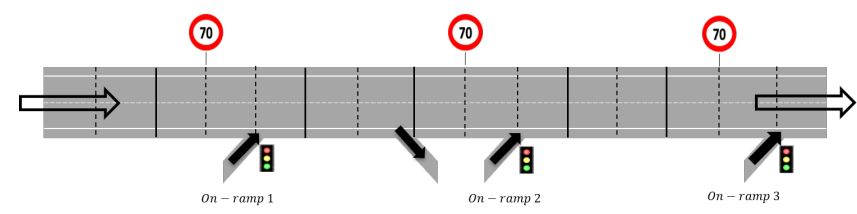

Fig. 4. Scenario scheme.

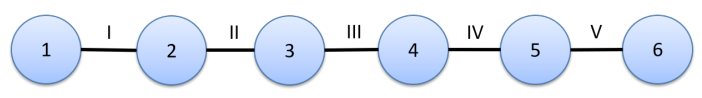

Fig. 5. Communication paths. 
TABLE I

Simulation AND MODEl PARAMETERS

\begin{tabular}{|c|c|c|c|}
\hline Symbol & Value & Symbol & Value \\
\hline$a$ & 1.867 & $\bar{T}$ & $10 \mathrm{~s}$ \\
\hline$\kappa$ & $40 \mathrm{veh} /(\mathrm{km} \cdot$ lane $)$ & $T_{\mathrm{u}}$ & $4 \min$ \\
\hline$\nu$ & $60(\mathrm{~km} \cdot \mathrm{km}) / \mathrm{h}$ & $T_{\text {top }}$ & $16 \mathrm{~min}$ \\
\hline$\tau$ & $18 \mathrm{~s}$ & $V S L_{\min }$ & $30 \mathrm{~km} / \mathrm{h}$ \\
\hline$\alpha$ & 1.05 & $V S L_{\max }$ & $100 \mathrm{~km} / \mathrm{h}$ \\
\hline$\lambda$ & 2 lanes & $r_{\min }$ & 0.3 \\
\hline$L$ & $1 \mathrm{~km}$ & $r_{\max }$ & 1 \\
\hline$\rho_{\text {crit }}$ & $33.5 \mathrm{veh} / \mathrm{km} / \mathrm{lane}$ & $\Delta_{\max }^{\mathrm{VSL}}$ & $20 \mathrm{~km} / \mathrm{h}$ \\
\hline$v_{\text {free }}$ & $102 \mathrm{~km} / \mathrm{h}$ & $\Delta_{\max }^{\mathrm{r}}$ & 0.5 \\
\hline$C_{\text {ramps }}$ & $2000 \mathrm{veh} / \mathrm{h}$ & $L_{\text {ramps }}$ & 100 veh \\
\hline$\beta_{m, i}$ & $\begin{array}{cc}0.1 & \text { if } m, i=3,2 \\
0 & \text { otherwise }\end{array}$ & $\epsilon$ & 0.02 \\
\hline
\end{tabular}

TABLE II

VARIABLE SPEED LIMITS AND ON/OFF-RAMPS IN THE STRETCH

\begin{tabular}{|l|r|r|}
\hline $\begin{array}{l}\text { VSL } \\
\text { Signal }\end{array}$ & $\begin{array}{c}\text { Starting } \\
\text { point }\end{array}$ & $\begin{array}{c}\text { Actuation } \\
\text { length }\end{array}$ \\
\hline \hline VSL 1 & $\mathrm{km} \mathrm{3}$ & $1 \mathrm{~km}$ \\
VSL 2 & $\mathrm{km} 8$ & $1 \mathrm{~km}$ \\
VSL 3 & $\mathrm{km} 13$ & $1 \mathrm{~km}$ \\
\hline
\end{tabular}

\begin{tabular}{|l|r|}
\hline $\begin{array}{l}\text { On/Off- } \\
\text { ramps }\end{array}$ & $\begin{array}{r}\text { Location } \\
\text { point }\end{array}$ \\
\hline \hline On-ramp 1 & $\mathrm{km} \mathrm{4}$ \\
On-ramp 2 & $\mathrm{km} \mathrm{9}$ \\
On-ramp 3 & $\mathrm{km} \mathrm{14}$ \\
Off-ramp 1 & $\mathrm{km} \mathrm{7}$ \\
\hline
\end{tabular}

is assumed to remain constant at the last measured value (see Assumption 3); (iii) the boundary conditions given in Subsection II.B(1) are applied accordingly to the corresponding substretch of road. Then, all variables that come into play in the coalitions' optimization problem belong to the cluster at issue, and all clusters can compute in parallel and independently their traffic signals. Also, notice that the partition imposed by the upper layer exploits coupling to foster the separability of the problem structure, i.e., very coupled stretches end up in the same coalition.

Remark 4: The simulations have been performed without model mismatch for simplicity. Hence, in this regards our results must be considered as a bound on the performance that can be achieved using this method. Also, note that the scope of the paper is to perform a first assessment of the proposed control strategy within this field while comparing its performance with other MPC formulations under the same conditions. Nevertheless, it must be noted that the MPC framework offers several possibilities to deal with model mismatches and other uncertainty sources, e.g., by using robust and stochastic MPC approaches.

Figures 6 shows the density evolution in the stretch for the no control case. We will work with two jams that have been intentionally induced in different sections of the road to see how the enabled links tend to arise in the most critical sections. The first peak of density is a consequence of both the mainstream flow and an increase in the number of cars arriving at the first on-ramp. The second one is equally caused by on-ramps 2 and 3 inflows. The mainstream demand has been set similarly as in [23]. That is, for the first two hours and a half, the inflow of vehicles at the origin takes the constant value $3500 \mathrm{veh} / \mathrm{h}$, and then drops until $1000 \mathrm{veh} / \mathrm{h}$ in approximately ten minutes. For the case of the on-ramps, periods of high demand at each of them have been simulated. For the rest of the time, the number of vehicles arriving at these entries remains at relatively low levels (under $300 \mathrm{veh} / \mathrm{h}$ ). Figure 7 shows the density evolution when the coalitional approach with a communication cost $c_{l}=4.5$ is implemented. In particular, the TTS went from $2793 \mathrm{veh} \cdot \mathrm{h}$ in the no control case to $2632 \mathrm{veh} \cdot \mathrm{h}$ in this example.

Some performance results are given in Table III. The results obtained with coalitional MPC are compared with decentralized MPC and with the performance obtained under a fully cooperative scheme, i.e., when all communication links remain enabled during the simulation. In particular, we have implemented the FC-MPC algorithm among all controllers with $N_{\mathrm{p}}=5 T_{u}$. In this table, apart from the TTS reductions, we indicate the waiting times on each on-ramp. In this respect, Figure 8 illustrates the queues of cars that were formed under the different control schemes. The flow that accesses the main road from on-ramp 1 is notably restricted, whose direct consequence is that mainstream density does not rise as in the no control case at expense of the queue length at on-ramp 1, which reaches the maximum value allowed after 0.60 hours of simulation. Additionally, waiting queues arise at on-ramp 2 and 3 but the impact of the second jam in the mainline is notably lessened.

In Figure 9, the communication links enabled over time for different communication costs $c_{l}$ are shown. In our example, the communication links commonly arise between a link with on-ramp and the one downstream. This is consistent with the definition of (25), as it fosters coalitions of controllers at the most congested sections (normally in the area of an on-ramp entry). In this respect, when the maximum density in the road is lower than $0.8 \rho_{\text {crit }}$ and there is no waiting queue with more than 20 veh, the system operates in a decentralized manner. Likewise, when communication costs in function (25) are greater than $\sum_{\ell \in \Lambda} J_{\ell}$, we prioritize the choice of $\Lambda=\emptyset$. The increase in $c_{l}$ has an impact on the global performance as expected. Figures 10(a) and 10(b) show the densities and mean speeds evolutions obtained for the case of decentralized MPC and two different communication costs.

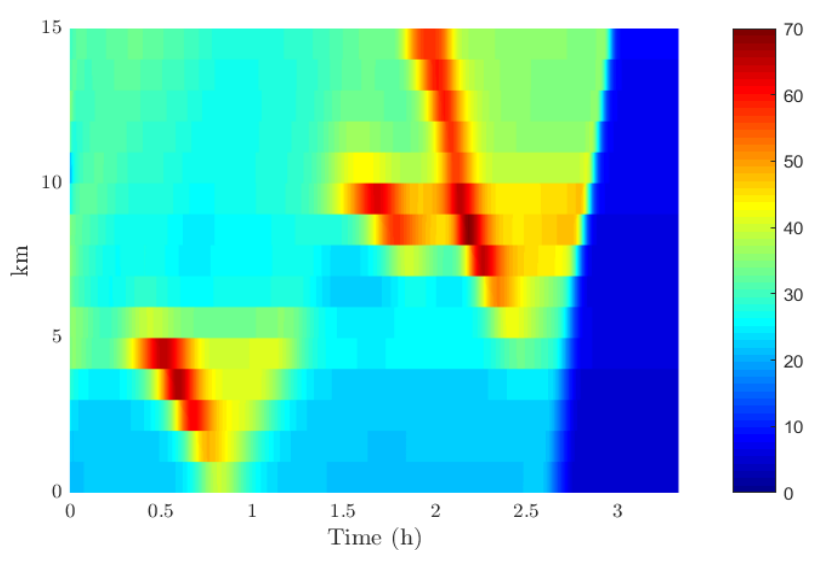

Fig. 6. Evolution of the densities $\rho_{m, i}[\mathrm{veh} / \mathrm{km}]$ in the stretch in the no control case. 


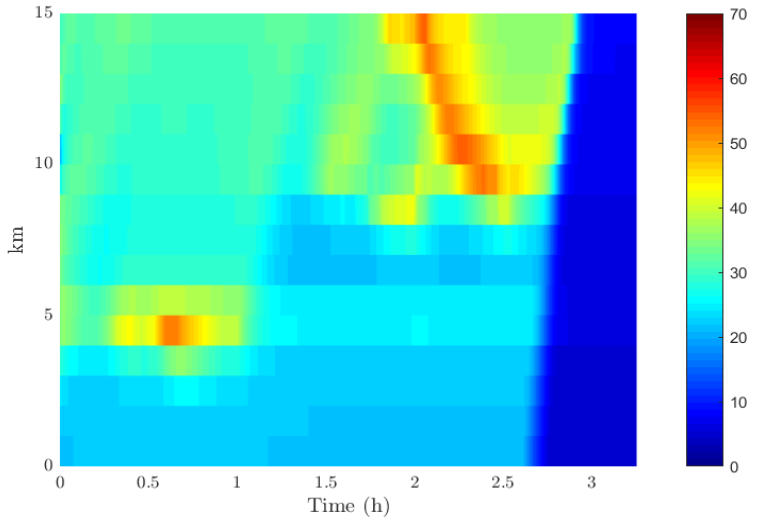

Fig. 7. Evolution of the densities $\rho_{m, i}[\mathrm{veh} / \mathrm{km}]$ in the stretch when using coalitional MPC with $c_{l}=4.5$.

TABLE III

SUMMARY OF PERFORMANCE RESULTS

\begin{tabular}{|c|c|c|c|c|c|c|}
\hline & \multirow{2}{*}{ Decent. } & \multicolumn{3}{|c|}{$c_{l}$} & \multirow{2}{*}{ FC-MPC } \\
\hline & & & 5.5 & 4.5 & 1 & \\
\hline \multicolumn{2}{|c|}{ TTS $(v e h \cdot h)$} & 2682 & 2647 & 2632 & 2608 & 2588 \\
\hline \multirow{3}{*}{ 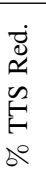 } & $\begin{array}{c}\text { Links } \\
1-3\end{array}$ & 8.90 & 10.60 & 10.77 & 10.49 & 9.71 \\
\hline & $\begin{array}{c}\text { Links } \\
4-6 \\
\end{array}$ & 2.09 & 2.91 & 5.40 & 7.94 & 10.23 \\
\hline & Global & 4.01 & 5.27 & 5.80 & 6.63 & 7.38 \\
\hline \multirow{3}{*}{ 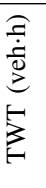 } & $\begin{array}{c}\text { On-ramp } \\
1\end{array}$ & 31.78 & 38.02 & 42.51 & 42.85 & 44.55 \\
\hline & $\begin{array}{c}\text { On-ramp } \\
2 \\
\end{array}$ & 0.52 & 0 & 18.79 & 34.77 & 34.66 \\
\hline & $\begin{array}{c}\text { On-ramp } \\
3\end{array}$ & 21.26 & 21.50 & 26.66 & 21.19 & 23.72 \\
\hline \multicolumn{2}{|c|}{ Comp. Time (min) } & 5.38 & 6.53 & 9.94 & 13.07 & 25.75 \\
\hline
\end{tabular}

Note that each subplot shows all segments in each freeway link. Additionally, Figure 10(c) illustrates the impact in the outflow of the network. In this case, for a better representation, we show the most representative intervals in the simulation. Finally, the control inputs, i.e., VSLs and on-ramp metering, are given in Figure 11, where it is shown that the VSLs tend to slow down the flow of vehicles arriving to the on-ramp areas during the periods of higher demand at these points. The decrease of $r_{3,2}$ at about $2.5 \mathrm{~h}$ does not have a significant effect on the vehicle's queue (see Figure 8) because the demand at on-ramp 2 is at a low-level during that part of the simulation (300 veh/h). However, a small improvement of the objective function is detected by the controllers if $r_{3,2}$ slightly restricts the access to the main road.

\section{CONCLUSION}

Coalitional MPC can certainly deal with freeways traffic problems and adapts naturally to the time-varying coupling conditions of this class of systems. The information exchange between local controllers in a distributed traffic system is crucial for its global performance. In this paper, it has been observed that the coalitional approach can provide a range of performance between decentralized and fully cooperative control schemes, while relieving the coordination requirements

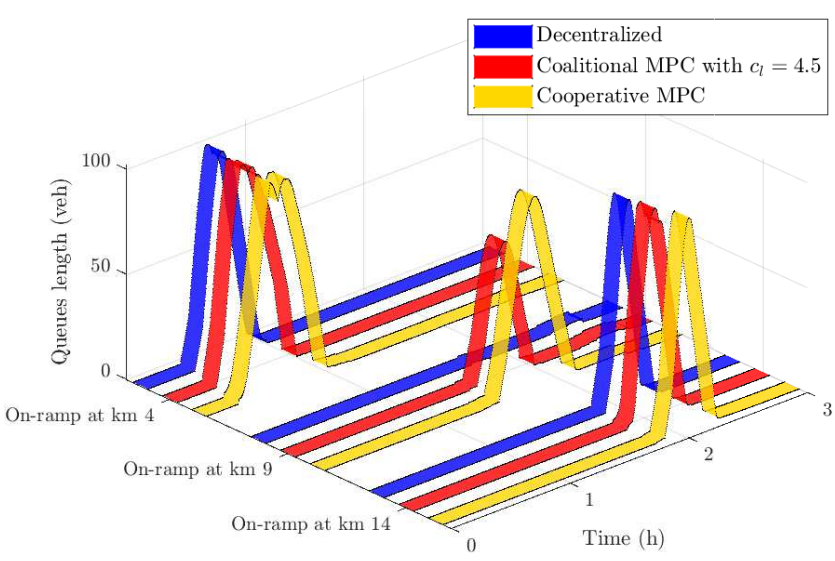

Fig. 8. Waiting queues $w_{m, i}$ at the on-ramps when the system is controlled in a decentralized manner, when using coalitional control with $c_{l}=4.5$, and when implementing a fully cooperative scheme.
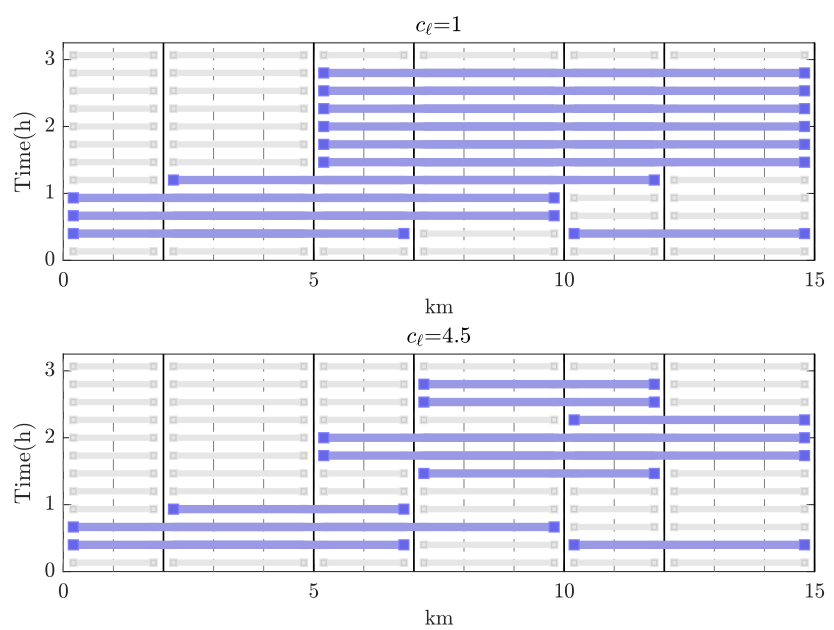

Fig. 9. Communication links enabled over time for different values of $c_{l}$.

associated with the latter. Increasing the outflow of a link when the downstream one is congested may result positive for the particular link but not at a network-level, and also represents a risk for traffic waves that can also propagate upstream. In this line, in case of a freeway link with an on-ramp close to its entrance, sharing information with the controller upstream is interesting, especially when the latter can govern the flow through variable speed limits, i.e., the agent in charge of the link with the on-ramp will ask the one upstream to decrease the VSL when its situation is congested or the queue is too long.

To illustrate the proposed approach, coalitional MPC has been simulated on a $15 \mathrm{~km}$ stretch of road. The performance of the global system improved even when just cooperation between two adjacent freeway links is considered, thus allowing us to notably reduce the complexity of the control architecture and to save communication and coordination costs. 

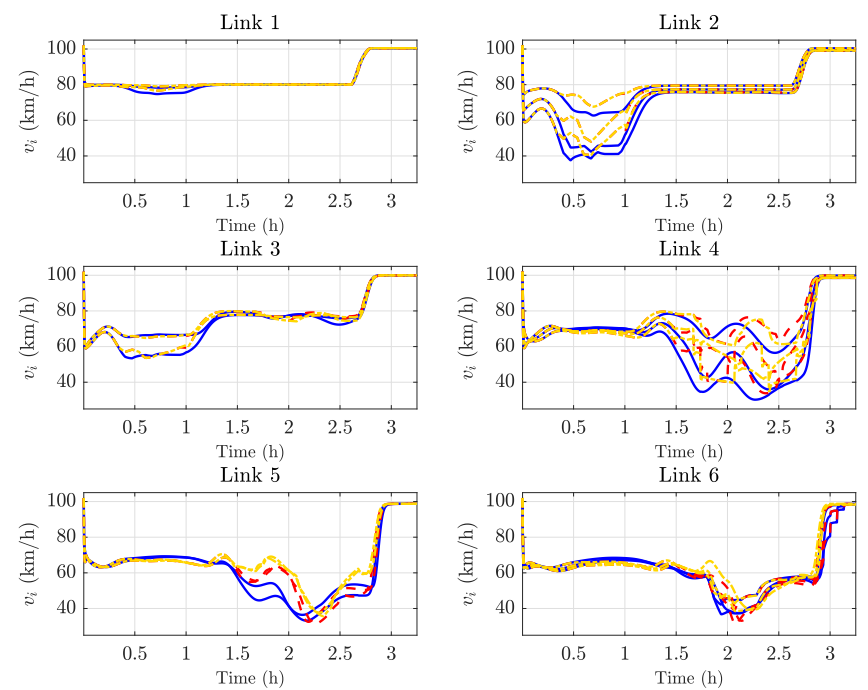

—Decentralized MPC - - - Coal. MPC $\left(c_{l}=4.5\right)-\cdots$ Coal. MPC $\left(c_{l}=1\right)$

(a) Mean speeds
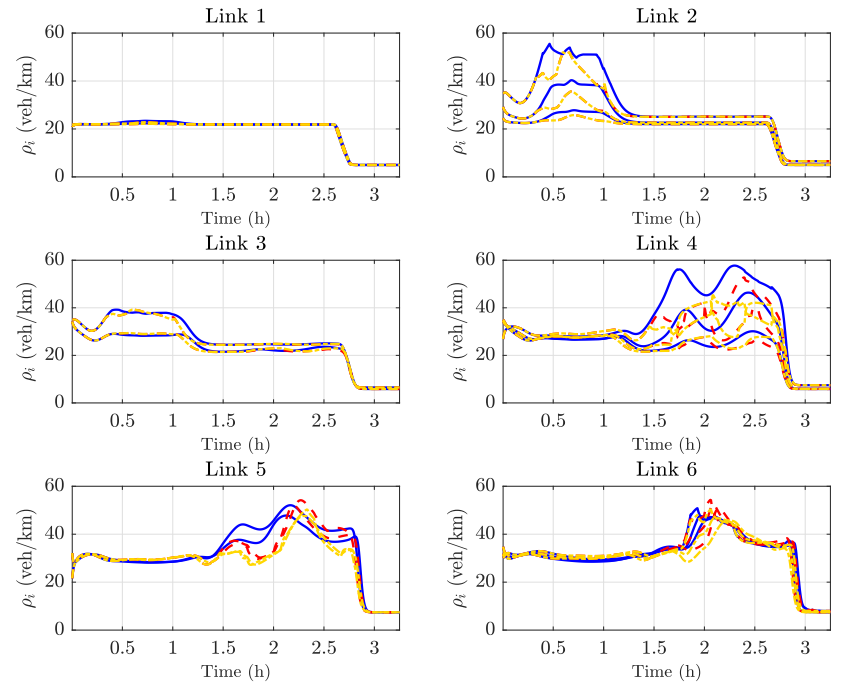

Decentralized MPC - - - Coal. MPC $\left(c_{l}=4.5\right)-\cdots$ Coal. MPC $\left(c_{l}=1\right)$

(b) Densities
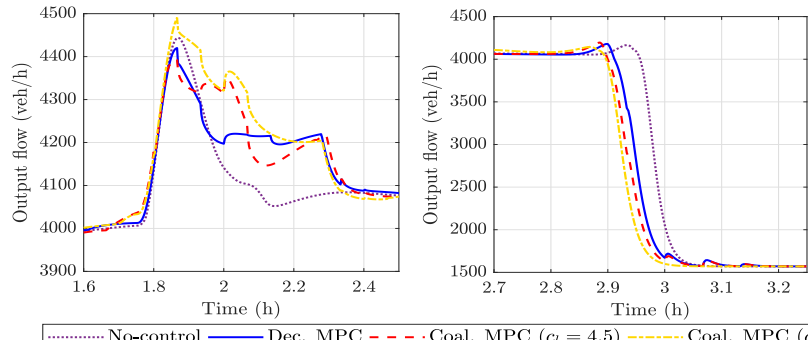

(c) Output flow

Fig. 10. Evolutions of the mean speeds $v_{m, i}$ (a), densities $\rho_{m, i}$ (b) and network outflow (c).
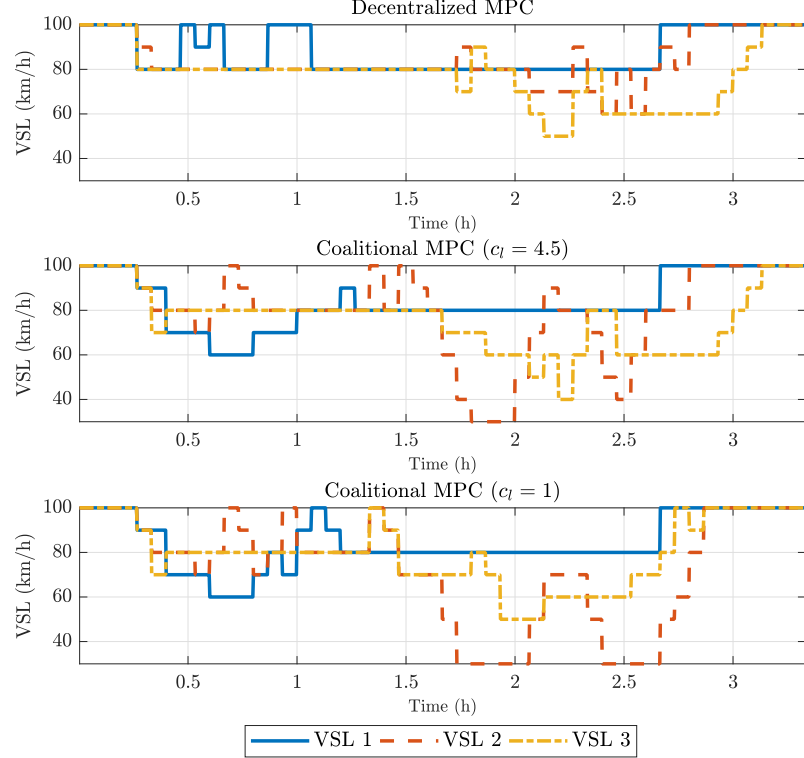

(a) Variable Speed Limits
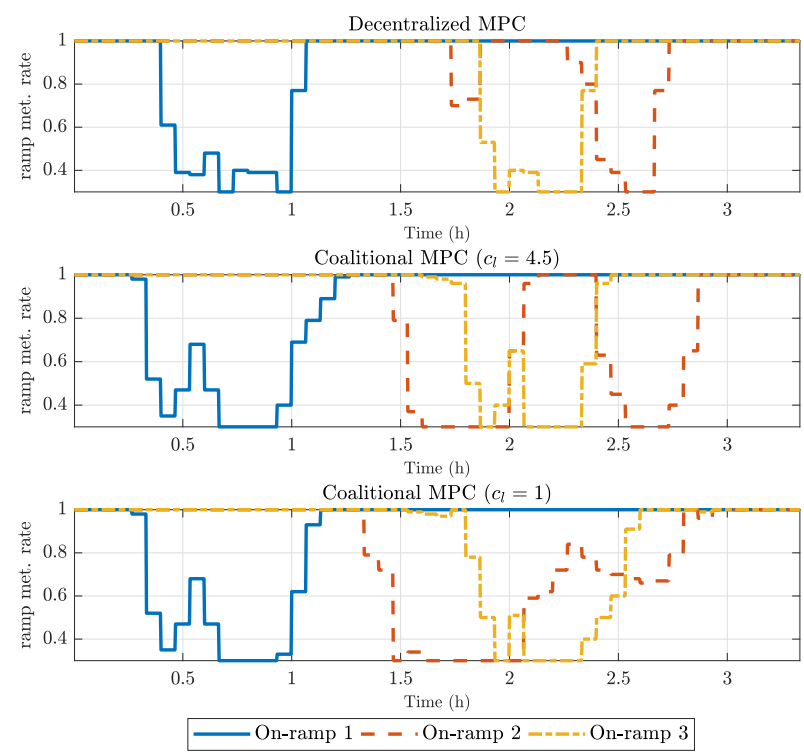

(b) On-ramp metering

Fig. 11. Evolution of the control actions.

\section{REFERENCES}

[1] E. F. Camacho and C. B. Alba, Model predictive control. Springer Science \& Business Media, 2013.

[2] A. Hegyi, B. De Schutter, and H. Hellendoorn, "Model predictive control for optimal coordination of ramp metering and variable speed limits," Transportation Research Part C: Emerging Technologies, vol. 13, no. 3, pp. 185-209, 2005.

[3] R. C. Carlson, I. Papamichail, M. Papageorgiou, and A. Messmer, "Optimal motorway traffic flow control involving variable speed limits and ramp metering," Transportation Science, vol. 44, no. 2, pp. 238-253, 2010.

[4] A. Muralidharan and R. Horowitz, "Computationally efficient model predictive control of freeway networks," Transportation Research Part C: Emerging Technologies, vol. 58, pp. 532 - 553, 2015, special Issue: Advanced Road Traffic Control. 
[5] M. Papageorgiou and A. Kotsialos, "Freeway ramp metering: An overview," IEEE transactions on intelligent transportation systems, vol. 3, no. 4, pp. 271-281, 2002.

[6] M. Papageorgiou, E. Kosmatopoulos, and I. Papamichail, "Effects of variable speed limits on motorway traffic flow," Transportation Research Record: Journal of the Transportation Research Board, no. 2047, pp. 37-48, 2008.

[7] J. R. D. Frejo, I. Papamichail, M. Papageorgiou, and E. F. Camacho, "Macroscopic modeling and control of reversible lanes on freeways," IEEE Transactions on Intelligent Transportation Systems, vol. 17, no. 4, pp. 948-959, 2016.

[8] A. Karimi, A. Hegyi, B. De Schutter, H. Hellendoorn, and F. Middelham, "Integration of dynamic route guidance and freeway ramp metering using model predictive control," in American Control Conference, 2004. Proceedings of the 2004, vol. 6. IEEE, 2004, pp. 5533-5538.

[9] P. D. Christofides, R. Scattolini, D. M. de la Peña, and J. Liu, "Distributed model predictive control: A tutorial review and future research directions," Computers \& Chemical Engineering, vol. 51, pp. 21-41, 2013.

[10] J. M. Maestre, R. R. Negenborn et al., Distributed model predictive control made easy. Springer, 2014, vol. 69.

[11] J. M. Maestre, D. M. De La Peña, E. F. Camacho, and T. Alamo, "Distributed model predictive control based on agent negotiation," Journal of Process Control, vol. 21, no. 5, pp. 685-697, 2011.

[12] J. M. Maestre, D. Muñoz de la Peña, A. Jiménez Losada, E. Algaba, and E. F. Camacho, "A coalitional control scheme with applications to cooperative game theory," Optimal Control Applications and Methods, vol. 35, no. 5, pp. 592-608, 2014.

[13] A. Nedić and D. Bauso, "Dynamic coalitional TU games: Distributed bargaining among players' neighbors," IEEE Transactions on Automatic Control, vol. 58, no. 6, pp. 1363-1376, 2013.

[14] M. Marzband, R. R. Ardeshiri, M. Moafi, and H. Uppal, "Distributed generation for economic benefit maximization through coalition formation-based game theory concept," International Transactions on Electrical Energy Systems, vol. 27, no. 6, p. e2313, 2017.

[15] F. J. Muros, J. M. Maestre, E. Algaba, T. Alamo, and E. F. Camacho, "Networked control design for coalitional schemes using game-theoretic methods," Automatica, vol. 78, pp. 320-332, 2017.

[16] T. Ishizaki, K. Kashima, J.-i. Imura, and K. Aihara, "Model reduction and clusterization of large-scale bidirectional networks." IEEE Trans. Automat. Contr., vol. 59, no. 1, pp. 48-63, 2014.

[17] A. Jain, A. Chakrabortty, and E. Biyik, "Distributed wide-area control of power system oscillations under communication and actuation constraints," Control Engineering Practice, vol. 74, pp. 132-143, 2018.

[18] Y. Zheng, Y. Wei, and $\mathrm{S}$. Li, "Coupling degree clustering-based distributed model predictive control network design," IEEE Transactions on Automation Science and Engineering, 2018.

[19] F. Lian, A. Chakrabortty, and A. Duel-Hallen, "Game-theoretic multiagent control and network cost allocation under communication constraints," IEEE Journal on Selected Areas in Communications, vol. 35, no. 2, pp. 330-340, Feb 2017.

[20] F. Fele, J. M. Maestre, and E. F. Camacho, "Coalitional control: Cooperative game theory and control," IEEE Control Systems, vol. 37, no. 1, pp. 53-69, 2017.

[21] F. Fele, J. M. Maestre, S. M. Hashemy, D. M. de la Peña, and E. F Camacho, "Coalitional model predictive control of an irrigation canal," Journal of Process Control, vol. 24, no. 4, pp. 314-325, 2014.

[22] I. Papamichail, A. Kotsialos, I. Margonis, and M. Papageorgiou, "Coordinated ramp metering for freeway networks-a model-predictive hierarchical control approach," Transportation Research Part C: Emerging Technologies, vol. 18, no. 3, pp. 311-331, 2010

[23] A. Hegyi, B. De Schutter, and J. Hellendoorn, "Optimal coordination of variable speed limits to suppress shock waves," IEEE Transactions on intelligent transportation systems, vol. 6, no. 1, pp. 102-112, 2005.

[24] A. H. Ghods, L. Fu, and A. Rahimi-Kian, "An efficient optimization approach to real-time coordinated and integrated freeway traffic control," IEEE Transactions on Intelligent Transportation Systems, vol. 11, no. 4, pp. 873-884, 2010.

[25] J. R. D. Frejo and E. F. Camacho, "Global versus local MPC algorithms in freeway traffic control with ramp metering and variable speed limits," IEEE Transactions on intelligent transportation systems, vol. 13, no. 4, pp. $1556-1565,2012$.

[26] D. Whitley, "A genetic algorithm tutorial," Statistics and computing, vol. 4, no. 2, pp. 65-85, 1994

[27] A. Messmer and M. Papageorgiou, "Metanet: A macroscopic simulation program for motorway networks," Traffic Engineering \& Control, vol. 31, no. 9, 1990.
[28] T. Bellemans, B. De Schutter, and B. De Moor, "Model predictive control for ramp metering of motorway traffic: A case study," Control Engineering Practice, vol. 14, no. 7, pp. 757-767, 2006.

[29] C. F. Daganzo, "The cell transmission model: A dynamic representation of highway traffic consistent with the hydrodynamic theory," Transportation Research Part B: Methodological, vol. 28, no. 4, pp. 269-287, 1994.

[30] A. Bemporad and M. Morari, "Control of systems integrating logic, dynamics, and constraints," Automatica, vol. 35, no. 3, pp. 407-427, 1999.

[31] G. Gomes and R. Horowitz, "Optimal freeway ramp metering using the asymmetric cell transmission model," Transportation Research Part C: Emerging Technologies, vol. 14, no. 4, pp. 244-262, 2006.

[32] P. Grandinetti, C. Canudas-de Wit, and F. Garin, "Distributed optimal traffic lights design for large-scale urban networks," IEEE Transactions on Control Systems Technology, no. 99, pp. 1-14, 2018.

[33] P. Grandinetti, C. C. de Wit, and F. Garin, "An efficient one-stepahead optimal control for urban signalized traffic networks based on an averaged cell-transmission model," in Control Conference (ECC), 2015 European. IEEE, 2015, pp. 3478-3483.

[34] A. N. Venkat, I. A. Hiskens, J. B. Rawlings, and S. J. Wright, "Distributed mpc strategies with application to power system automatic generation control," IEEE transactions on control systems technology, vol. 16, no. 6, pp. 1192-1206, 2008.

[35] S. Riverso, F. Boem, G. Ferrari-Trecate, and T. Parisini, "Plug-and-play fault detection and control-reconfiguration for a class of nonlinear largescale constrained systems," IEEE Transactions on Automatic Control, vol. 61, no. 12, pp. 3963-3978, 2016.

[36] A. Zakharov, E. Zattoni, M. Yu, and S.-L. Jämsä-Jounela, "A performance optimization algorithm for controller reconfiguration in fault tolerant distributed model predictive control," Journal of Process Control, vol. 34, pp. 56-69, 2015.

[37] D. Chilin, J. Liu, J. F. Davis, and P. D. Christofides, "Data-based monitoring and reconfiguration of a distributed model predictive control system," International Journal of Robust and Nonlinear Control, vol. 22, no. 1, pp. 68-88, 2012.

[38] J. M. Maestre, P. A. Trodden, and H. Ishii, "A distributed model predictive control scheme with robustness against noncompliant controllers," in 2018 IEEE Conference on Decision and Control (CDC). IEEE, 2018, pp. 3704-3709.

[39] F. J. Muros, J. M. Maestre, C. Ocampo-Martinez, E. Algaba, and E. F. Camacho, "A game theoretical randomized method for large-scale systems partitioning," IEEE access, vol. 6, pp. 42 245-42 263, 2018.

[40] L. A. Fletscher, J. M. Maestre, and C. V. Peroni, "Coalitional planning for energy efficiency of hetnets powered by hybrid energy sources," IEEE Transactions on Vehicular Technology, vol. 67, no. 7, pp. 65736584, 2018.

[41] J. Castro, D. Gómez, and J. Tejada, "Polynomial calculation of the shapley value based on sampling," Computers \& Operations Research, vol. 36 , no. 5, pp. 1726-1730, 2009.

[42] J. M. Maestre and H. Ishii, "A pagerank based coalitional control scheme," International Journal of Control, Automation and Systems, vol. 15, no. 5, pp. 1983-1990, 2017.

[43] H. Ishii and R. Tempo, "Distributed randomized algorithms for the pagerank computation," IEEE Transactions on Automatic Control, vol. 55, no. 9, pp. 1987-2002, 2010.

[44] H. Ceylan and M. G. Bell, "Traffic signal timing optimisation based on genetic algorithm approach, including drivers' routing," Transportation Research Part B: Methodological, vol. 38, no. 4, pp. 329-342, 2004.

[45] F. Teklu, A. Sumalee, and D. Watling, "A genetic algorithm approach for optimizing traffic control signals considering routing," Computer-Aided Civil and Infrastructure Engineering, vol. 22, no. 1, pp. 31-43, 2007.

[46] R. Putha, L. Quadrifoglio, and E. Zechman, "Comparing ant colony optimization and genetic algorithm approaches for solving traffic signal coordination under oversaturation conditions," Computer-Aided Civil and Infrastructure Engineering, vol. 27, no. 1, pp. 14-28, 2012.

[47] J. R. D. Frejo, A. Núñez, B. De Schutter, and E. F. Camacho, "Hybrid model predictive control for freeway traffic using discrete speed limit signals," Transportation Research Part C: Emerging Technologies, vol. 46, pp. 309-325, 2014.

[48] A. Kotsialos, M. Papageorgiou, and A. Messmer, "Optimal coordinated and integrated motorway network traffic control," in 14th International Symposium on Transportation and Traffic TheoryTransportation Research Institute, 1999.

[49] X.-Y. Lu, P. Varaiya, R. Horowitz, D. Su, and S. Shladover, "Novel freeway traffic control with variable speed limit and coordinated ramp metering," Transportation Research Record: Journal of the Transportation Research Board, no. 2229, pp. 55-65, 2011. 


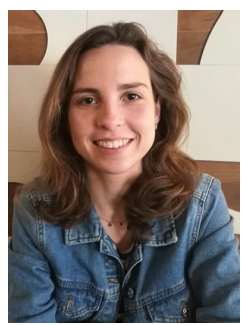

Paula Chanfreut received her Master degree in Industrial Engineering by the University of Seville, Spain, in 2019. Currently, she is a pre-doctoral fellow under the University Professor Training Program (FPU) at the Dept. of Systems and Automation Engineering, University of Seville, under the supervision of prof. E. F. Camacho and J. M. Maestre. Her research interests are in the area of distributed control for networked systems. She can be contacted at Departmento de Ingeniería de Sistemas y Automática, ETSI Universidad de Sevilla, Camino de los Descubrimientos, s/n. 41092 Sevilla, Spain.

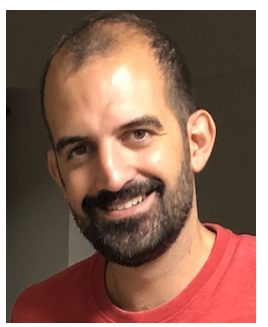

José M. Maestre received the $\mathrm{PhD}$ on automation and robotics by the University of Seville, where he works as associate professor. He has also worked in LTH at Lund University (guest researcher), TU Delft (postdoc), University of Cadiz (assistant professor), University of Pavia (visiting professor), and Tokyo Institute of Technology (overseas researcher). Besides his $\mathrm{PhD}$, which was awarded with the extraordinary prize of the University of Seville, he has master degrees on Smart Homes and Intelligent Buildings (Technical University of Madrid), Telecommunications Economics (National Distance Education University), Economics and Development (University of Seville), and Model-based Drug Development (University of Manchester). His main research interests are the control of distributed systems, the integration of service robots in the smart home, and the applications of control to cyber-physical systems. He has authored and coauthored more than one hundred journal and conference papers regarding these topics. Also he has (co)edited the books Service robotics within the Digital Home: Applications and Future Prospects (Springer 2011), Distributed Model Predictive Control Made Easy (Springer, 2014) and Domótica para Ingenieros (Paraninfo, 2015), and he has (co)authored several books, including A Programar se Aprende Jugando (Paraninfo, 2017) and Sistemas de Medida y Regulación (Paraninfo, 2018) . Finally, he has been involved in the development of several technological firms such as Idener and Mobile Water Management.

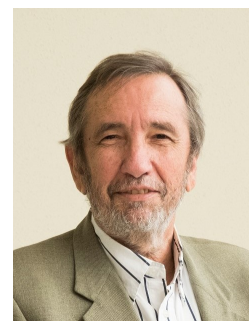

Eduardo F. Camacho received the doctorate in electrical engineering from the University of Seville, Spain, where he is now a professor in the Department of System Engineering and Automatic Control. He has written several books, including Model Predictive Control in the Process Industry (Springer- Verlag, 1995), Advanced Control of Solar Plants (Springer-Verlag, 1997), Model Predictive Control (Springer-Verlag, 1999, 2004 second edition), Control e Instrumentacion de Procesos Químicos (Ed. Sintesis), Control of Dead-Time Processes (Springer-Verlag, 2007), and Control of Solar Systems (SpringerVerlag, 2011, 2015 Chinese translation). He chaired the IFAC Publication Committee and the IFAC Policy Committee. He was the president of the European Union Control Association and chaired the IEEE Control Systems Society (CSS) International Affairs Committee. He was a member of the IEEE CSS Board of Governors and he is currently a member of IFAC Council. He is a Fellow of the IEEE and IFAC. He was awarded an Advanced Grant by the European Reseach Council in 2018. 